\title{
Adopción de tecnología de conservación de suelos y agua y su efecto en los ingresos agropecuarios y el contenido de materia orgánica del suelo
}

\section{Adoption of soil and water conservation technologies and its effect on agricultural incomes and organic matter content}

\author{
Oswaldo Martin Pineda Rizo ${ }^{1}$, Fidel Guzmán-Guillen ${ }^{2}$ \\ 'Ingeniero agrónomo, MSc. en Desarrollo Rural, tierrapnd1@gmail.com \\ ${ }^{2}$ Dr. en planificación y gestión de proyectos del desarrollo rural sostenible, fidelblue@gmail.com \\ Facultad de Desarrollo Rural, Universidad Nacional Agraria, km 12 1⁄2 Carretera Norte. Managua, Nicaragua
}

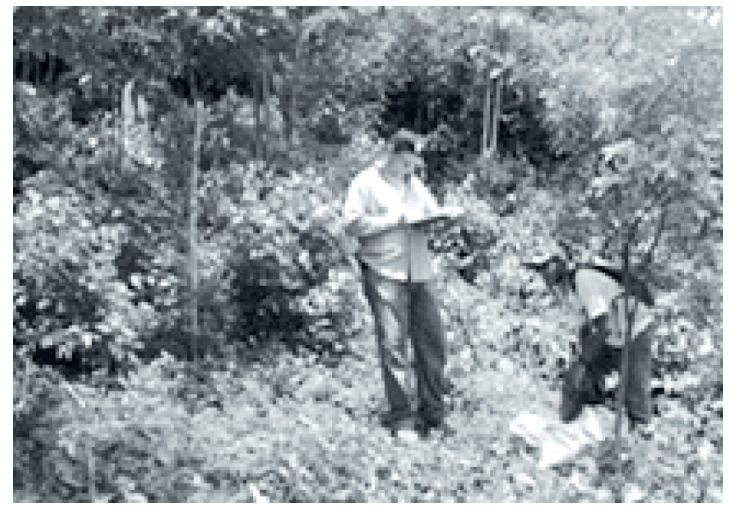

\section{RESUMEN}

Según el Instituto Interamericano de Cooperación para la Agricultura, en Nicaragua unos principales problemas de transferencia es la ausencia de indicadores de adopción de tecnología en conservación de suelo y agua. Debido a esto el estudio se da con la finalidad de documentar efectos ambientales y socio económicos a largo plazo del proceso de adopción de técnicas de conservación de suelos promovidas por las entidades Instituto Nicaragüense de Tecnología Agropecuario (INTA) y la Fundación de Investigación y Desarrollo Rural (FIDER) en los años 90s. El Titulo de la investigación es La adopción tecnológica en conservación de suelos y agua y su efecto en los ingresos agropecuarios y contenidos de materia orgánica en localidades rurales, 2002 al 2008, se ejecutó con una población muestral del $45 \%$, el proceso metodológico inicio con la fase de planeación para diseñar los instrumentos de campo y seleccionar la muestra por comunidad, seguido del proceso de Recolección de datos en campos, para luego ser analizados e interpretados con los comunitarios. Las variables evaluadas; (Adopción, Ingresos Productivos, Umbral de Reproducción Simple y Contenido de materia orgánica en los suelos). Los productores propietarios de tierra lograron adoptar hasta 16 técnicas de conservación y los no propietarios máximos 4 tecnologías. Las barreras vivas y muertas fueron las técnicas más adoptadas en las dos comunidades y los productores organizados fueron los más adoptadores. Los Ingresos se incrementan por encima del $50 \%$ a medida que se adoptan más tecnologías y se aproximan al Umbral de Reproducción Simple. Los contenidos de Materia orgánica se incrementan hasta $2.8 \%$ en sistemas combinados y con mayor tiempo de adopción.

Palabras clave: tecnología, extensión, fertilidad y calidad de suelo.

\begin{abstract}
According to the Inter-American Institute for Cooperation on Agriculture, in Nicaragua, the main transfer problems are the lack of technology adoption indicators for soil and water conservation. Due to this, the study is given with the purpose of documenting longterm environmental and socio-economic effects of the process of adoption of soil conservation techniques promoted by the Nicaraguan Institute of Agricultural Technology (INTA) and the Rural Research and Development Foundation (FIDER) in the 1990s. The title of the research is Technological adoption in soil and water conservation and its effect on agricultural income and organic matter contents in rural localities, from 2002 to 2008, was executed with a sample population of $45 \%$, the methodological process started with The planning phase to design the field instruments and select the sample per community, followed by the data collection process in fields, to be analyzed and interpreted with the community. The variables evaluated; (Adoption, Productive Income, Simple Reproduction Threshold and Organic matter content in soils). Land-owning producers were able to adopt up to 16 conservation techniques and maximum no-owners 4 technologies. The living and dead barriers were the most adopted techniques in the two communities and the organized producers were the most adopters. Revenues increase by more than $50 \%$ as more technologies are adopted and approach the Simple Playback Threshold. Organic matter contents increase to $2.8 \%$ in combined systems and with a longer adoption time. Keywords: Technology, extension, fertility and soil quality.
\end{abstract}


$\mathrm{U}$ nos de los principales problemas en la transferencia de tecnología de manejo sostenible de suelo y agua (MSSA) en Nicaragua desde los años 70, fue la falta de indicadores que permitieran poder medir la calidad de establecimiento y las estrategias de comunicación durante el proceso de aceptación y adopción por parte de los agricultores (IICA, 2005). En la actualidad no se cuentan con estudios de evaluación ex antes y ex post proyecto que sistematice experiencias con aquellas técnicas que se adoptaron exitosamente para ser replicadas, por tanto se plantea en conjunto con el Instituto Nicaragüense de Tecnología Agropecuaria (INTA) la necesidad de la realización de estudios de adopción y poder determinar si ha habido alguna contribución de la adopción en la mejora de los ingresos agropecuarios y en la calidad del suelo. El trabajo de investigación analizó los factores que intervienen en los procesos de adopción de tecnología de conservación de suelo y agua. Además de conocer el efecto que tienen la implementación de estas técnicas en la calidad de suelo y su contribución directa en la mejora de los ingresos productivo de aquellas familias. El objetivo general fue analizar los factores que inciden en la adopción de técnicas de conservación de suelo y agua, la contribución en los contenidos de materia orgánica del suelo e ingresos agropecuarios en las familias de las comunidades de Tomabú de La Trinidad y Los Pochotillos de San Francisco Libre en el periodo 2002 al 2008.

El INTA y la Fundación Integral para el Desarrollo Rural(FIDER) han promovido las técnicas de conservación de suelo y agua, desde inicio y mediado de los años noventas en zonas de laderas vulnerables a problemas de erosión hídrica, donde se obtiene la mayor parte de la producción de granos básicos. Estas instituciones transfieren y brindan asistencia técnica a productores para que implementen obras y prácticas conservacionistas con el objetivo de disminuir la perdida de los suelos, aumentar la fertilidad y los rendimientos en los cultivos. Sin embargo, no todos los pequeños productores han logrado adoptar las tecnologías. Se estima que la erosión actual ha alcanzado niveles de deterioro alarmantes ya que de las 7.7 millones de hectáreas no cubiertas de bosques, el $48.3 \%$ presenta erosión moderada a severa con pérdidas de espesor de suelo que varían de 20 hasta $65 \mathrm{~cm}$ en los casos más severos. Además de las estimaciones mencionadas, existen diversos resultados experimentales que confirman la magnitud que ha alcanzado la erosión a nivel nacional (IICA, 2005).

Según Barreto (1996), el $44 \%$ de las tierras de Nicaragua poseen pendientes mayores del $20 \%$, que están potencialmente expuestas a la erosión. La importancia radica que en estos suelos de laderas se obtiene el $79 \%$ de la producción de maíz y un $90 \%$ de la producción de frijol.
La importancia de este estudio se arraiga en evaluar los factores que influyen para que los productores o familias adoptan o no este tipo de tecnología y poder determinar si en aquellas fincas que tienen más de tres años de trabajar con tecnologías de conservación de suelos y agua (TCSA) existen efectos positivos y una contribución directa en los ingresos de los pequeños y medianos productores. Estudios realizados por PASOLAC (2003) muestra que en tres países de Centroamérica (Nicaragua, Honduras y Salvador) los productores trabajan con la técnica de manejos sostenible de suelo y agua (MSSA) lo que ha incrementado los rendimiento, presentando un promedio en los tres países entre $20-23.88$ qq ha-1 (909-1085 $\left.\mathrm{kg} \mathrm{ha}^{-1}\right)$ de maíz y entre $7.05-13.02 \mathrm{qq}$ $\mathrm{ha}^{-1}\left(320-592 \mathrm{~kg} \mathrm{~h}^{-1}\right)$ de frijol, teniendo un incremento en ingresos por manzana en Nicaragua que superan los USD 30.00 (PASOLAC, 2003).

\section{MATERIALES Y MÉTODOS}

Área de estudio: El estudio se realizó en las comunidades de Tomabú del Municipio de La Trinidad departamento de Estelí y Los Pochotillos del municipio de San Francisco Libre, Jurisdicción del departamento de Managua.

La Trinidad: Se encuentra localizada a $116 \mathrm{~km}$ al norte del municipio de Managua, capital de Nicaragua, (figura 1). Presenta una extensión territorial de $261 \mathrm{~km}^{2}$ y se ubica a $18^{\circ} 58^{\prime}$ de latitud norte y a $86^{\circ} 14^{\prime}$ de latitud oeste a una altura de $601.22 \mathrm{msnm}$. El Municipio comprende una población total de 23882 habitantes, con una distribución demográfica en el sector rural de 13782 habitantes y 10100 en área urbana (INIFOM, 2000).

San Francisco Libre: Se encuentra localizada a $79 \mathrm{~km}$ al noreste del municipio de Managua, capital de Nicaragua (figura 2), presenta una extensión territorial de $756 \mathrm{~km}^{2}$ y se ubica a los $12^{\circ} 30^{\prime}$ de latitud norte y $85^{\circ} 18^{\prime}$ de latitud oeste a una altura de $40 \mathrm{msnm}$. El Municipio comprende una población total de 10503 habitantes, con una distribución demográfica en el sector rural de 7423 habitantes y 3080 en área urbana (INIFOM, 2000).

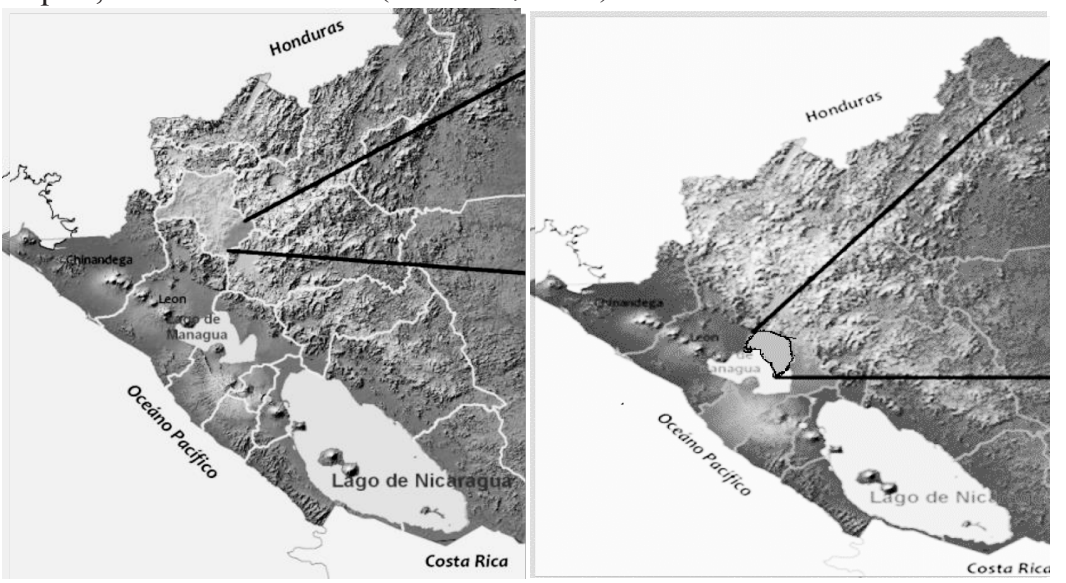

Figura 1. Ubicación del municipio La Trinidad, Estelí (izquierda) y San Francisco Libre, Managua (derecha). 
Descripción metodológica. Es una investigación aplicada del tipo no experimental, debido a que no hay manipulación de las variables y se fundamentó en el análisis de la realidad de una situación determinada. Según UNA (2008) en el documento guías y normas metodológicas de las formas de culminación de Estudio de la Universidad Nacional Agraria, posee un diseño del tipo descriptivo que está enfocado en las propiedades de los objetos o de las situaciones.

El estudio se desarrolló en cuatro fases metodológicas: fase I: organización y revisión de fuentes bibliográficas, fase II: diseño y validación del instrumento de campo, fase III: Recolección de datos, análisis y procesamiento y fase IV: socialización.

La muestra utilizada es del tipo razonada donde los elementos o sujetos elegidos cumplieron con requisitos, útiles para obtener los datos de investigación. La muestra representa el $45 \%$ de la población total, definiendo los siguientes criterios: a) productores que han sido atendidos por instituciones que han trabajado en técnicas en conservación de suelos y agua, en la zona de estudio durante el período 2002 al 2008). Productores con área entre 0.35 y 7.02 ha (0.5 y $10 \mathrm{Mz})$ de 7.03 a $35.13 \mathrm{ha}(10.01$ a $50 \mathrm{Mz})$, de 35.14 hasta 70.27 ha $(50.01$ hasta $100 \mathrm{Mz})$ y de 70.28 hasta 140.55 ha $(100.01$ a $200 \mathrm{Mz})$, c) productores que han recibido capacitación y asistencia técnica en obras de conservación de suelos y agua, en la zona de estudio durante el período señalado $\mathrm{y} d$ ) productores que cultivan granos básicos $\mathrm{y}$ hortalizas.

Para el cumplimiento de los objetivos propuestos se seleccionaron las siguientes variables: adopción, ingresos agropecuarios, umbral de reproducción simple y nivel de materia orgánica del suelo.

Los resultados manifestados se obtuvieron a través de un análisis comparativo de las dos zonas de estudio, que permitió identificar aquellos factores que inciden en los procesos de adopción y su efecto que tienen las TCSA adoptada, en la calidad de los suelos y la mejoría de los ingresos productivos.

\section{RESULTADOS Y DISCUSIÓN}

Miembros de familias productoras y su relación con la adopción de TCSA. Según la guía técnica de conservación de suelo y agua PASOLAC (1999). La disponibilidad de mano de obra es uno de los factores más importantes que determina la capacidad del productor de adoptar TCSA. Por ejemplo, la alta cantidad de mano de obra necesaria para la construcción de barreras muertas o el mantenimiento de los cultivos en callejones con sus podas frecuentes, afectan la difusión de estas prácticas en fincas con una escasez de mano de obra.

En Tomabú el promedio de miembros por familia es de 5.39 y el promedio de adopción por familia es de 4.74, en cambio en Pochotillos es de 4.71 en el caso de miembros por familia y de 3.21 las tecnologías adoptadas. Estos resultados indican que donde hay mayor cantidad de fuerza laboral o disponibilidad de mano de obra familiar, el número de adopción de las técnicas de conservación de suelo y agua se incrementa.

Adopción de TCSA según nivel de escolaridad. Unos de los factores que inciden en la adopción de tecnologías es el nivel de educación. Rogers (1995), expresa que las personas que presenten mayores dificultades para la apropiación de la información, cuentan con menos herramientas que le permita tomar decisiones en la adopción.

La mayoría de las familias tienen un nivel de educación primaria en ambas comunidades, siendo éste el grupo que presenta mayor números de técnicas de conservación de suelo y agua adoptadas. En Tomabú, la educación secundaria es el otro colectivo principal de adopción sumado a las vías de acceso. Otro aspecto importante es el número de técnicas adoptadas por parte de personas que no tienen ningún nivel de escolaridad, representando el $23 \%$ en el caso de Pochotillos y el 33\% para Tomabú. Esto refleja como el nivel de escolaridad limita la adopción (figura 2).

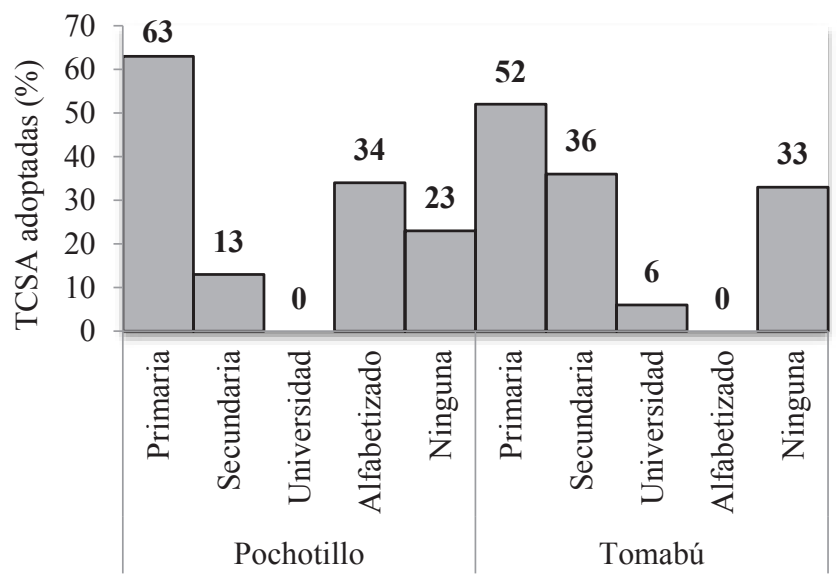

Figura 2. Nivel de adopción según nievl de escolaridad Porcentaje de productores según nivel de escolaridad y número de técnicas adoptadas.

Capacitación y adopción. La función esencial en las actividades de extensión es la de capacitar y transferir tecnologías o enseñar a los productores, con el fin de conseguir el máximo de transmisión de información, conocimiento y adopción de la tecnología propuesta.

La figura 3, detalla las capacitaciones recibidas, de ellas en tres temas hubo una mayor participación por parte de los productores, siendo barreras vivas (Bv), barreras muertas (Bm) y curvas a nivel (Can). En el caso de Tomabú, curvas a nivel fue el tema de capcitación con mayor participación de productores así como una de las adoptadas. En Los Pochotillos este mismo comportamiento se obtuvo con $\mathrm{Bv}$ y $\mathrm{Bm}$, donde hubo mayor asistencia reflejando mayor adopción por parte de los productores. Podemos decir que la capacitación influye en la adopción de tecnologías. 


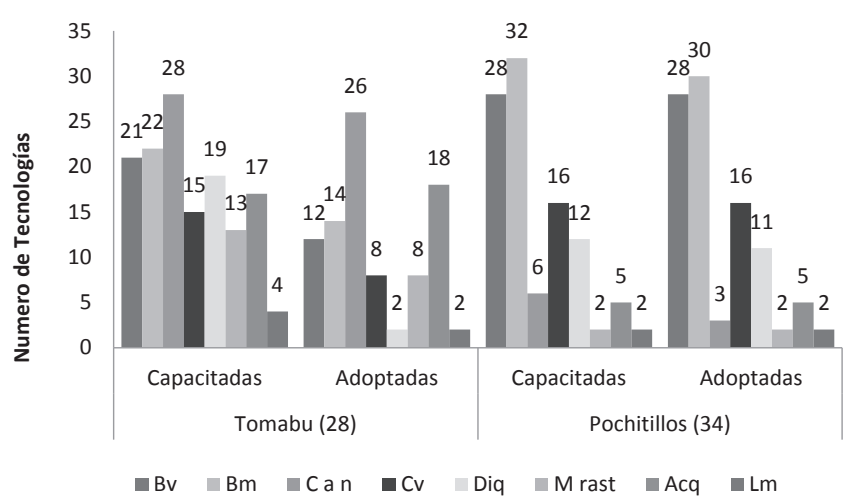

Figura 3. Numero de técnicas adoptadas según capacitación recibida por comunidad.

Cv: Cercas vivas, Diq: diques, $M$ rast: manejo de rastrojos, Acq: acequias, Lm: labranza mínima.

Organización y adopción de TCSA. Con los procesos de organización se ha logrado aumentar la participación de los productores, permitiendo una mayor y mejor divulgación de los resultados obtenidos; lo que facilita la adopción de las nuevas tecnologías (Hernández y Araya, 2004).

Se hizo evidente la tendencia de mayor adopción de tecnologías de conservación de suelo y agua en los sistemas de producción de productores organizados, $68 \%$ en el caso de Tomabú y 91\% en Pochotillos.

Ingresos y adopción. PASOLAC (2003), manifiesta que hay mejoría de ingresos en aquellos productores que cultivan en áreas con manejo sostenible de suelo y agua (MSSA). En este estudio se identofocó que la distribución porcentual de los ingresos promedios anuales provenientes de áreas productivas con TCSA es similar en ambas zonas, siendo de $63 \%$ en Los Pochotillos y de $62 \%$ en Tomabú. En el caso de áreas productivas sin TCSA en Los Pochotillos es de $37 \%$ y en Tomabú de 38\%.

Umbral de reproducción simple (URS) y adopción. Estudios realizados por Reyes y Arauz, (2009) determinaron que los ingresos económicos no es un factor incidente para la adopción de tecnologías de conservación de suelo y agua, sin embargo, tanto en Tomabú como en Los Pochotillos se registra que a mayor número de técnicas de conservación de suelos y agua, mayor es el aporte en los ingresos productivos (figuras 4 y 5) Para el caso de Los Pochotillos los ingresos aumenta cuando la adopción superas las ocho TCSA.

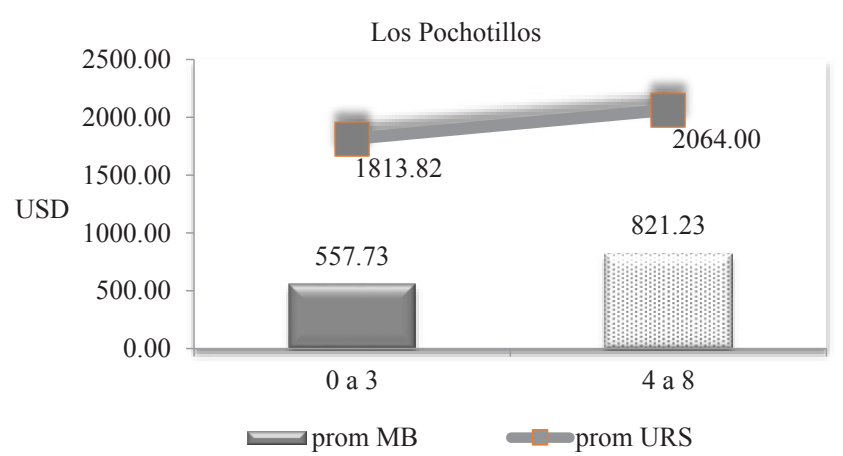

Figura 4. Relación margen bruto, URS y $\mathrm{N}^{\circ} \mathrm{TCSA}$ adoptadas por comunidad.

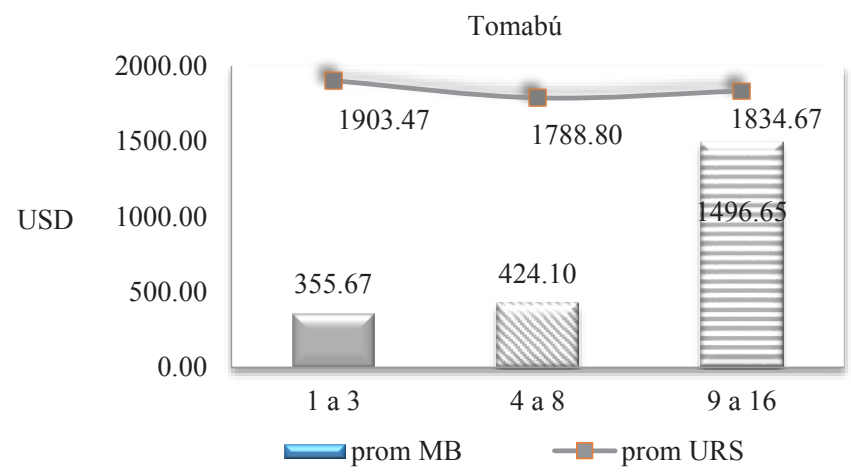

Figura 5. Relación margen bruto, URS y $\mathrm{N}^{\circ}$ de TCSA adoptadas por comunidad.

Materia orgánica y adopción. Aunque la materia orgánica constituye una pequeña fracción de la mayoría de los suelos, es un componente dinámico que ejerce una influencia dominante en muchas propiedades y procesos del suelo. Frecuentemente un efecto lleva a otro, de modo que de la adición de materia orgánica a los suelos, resulta en una cadena compleja de múltiples beneficios (Kononova, 1981).

Los resultados del contenido de materia orgánica presentados en la figura 6 reflejan dos umbrales como comparadores extremos, uno de ellos realizados en parcela agrícola sin ningún manejo de conservación y el otro bajo un sistema de bosque. Los análisis muestran un incremento gradual de los contenidos de materia orgánica para la comunidad de Tomabú, conforme al avance de los años de implementación y establecimiento de las técnicas de conservación de suelo y agua, llegando a aumentar hasta en $1.73 \%$ los contenidos de materia orgánica. 


\section{DESARROLLORURAL}

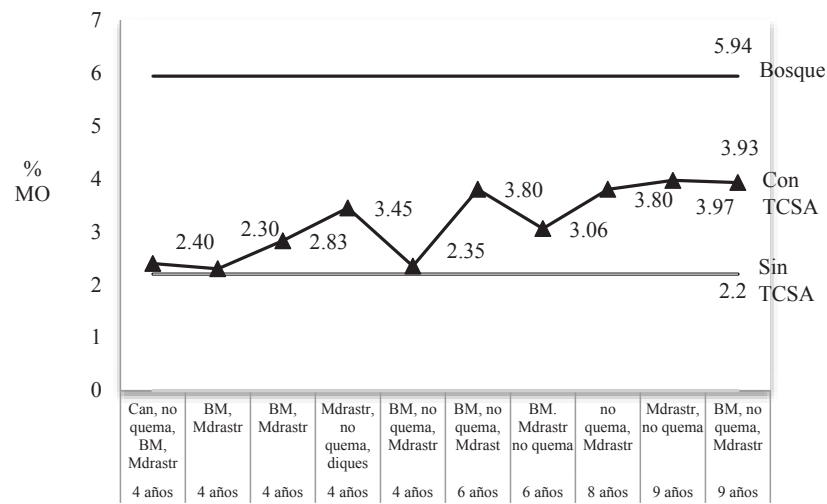

Figura 6. Comportamiento de la materia orgánica según TCSA, período de establecimiento y comparadores extremos, Tomabú.

En la figura 7 se indican los contenidos de materia orgánica la comunidad Los Pochotillos. Al hacer uso de dos comparadores extremos, uno de ellos referido a parcela agrícola sin manejo de conservación y el otro un sistema de bosques se observa que después de cinco años de adopción de técnicas de conservación de suelo y agua, se obetienen valores de $4.3 \%$ de materia orgánica, valor clasificado como, representando un incrmento de $2.8 \%$.

\section{CONCLUSIONES}

El proceso de adopción de tecnologías de conservación en las comunidades de Los Pochotillos y Tomabú se ve favorecido por la disponibilidad mano de obra familiar, organización comunitaria y por la escolaridad.

La adopción de sistemas de conservación de suelo

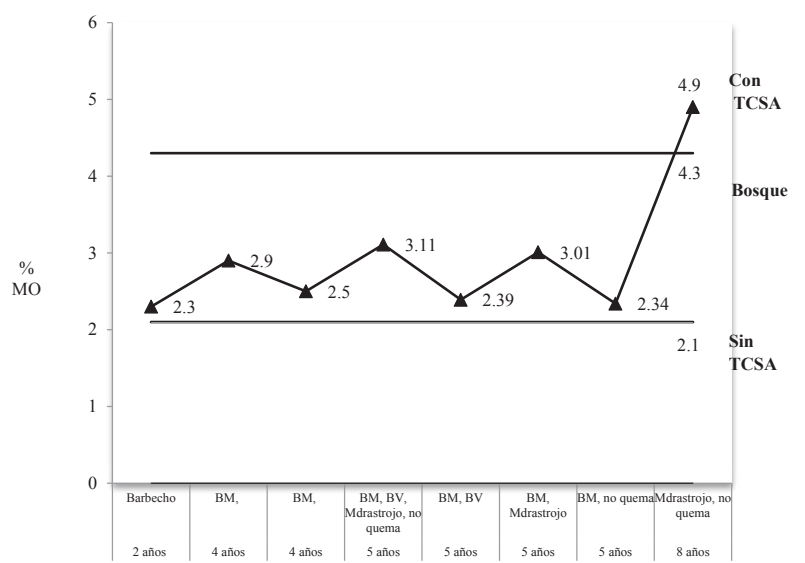

Figura 7. Comportamiento de la materia orgánica según TCSA, período de establecimiento y comparadores extremos, Los Pochotillos.

combinado a largo plazo contribuye hasta en un $60 \%$ de los ingresos en la unidad productiva y si se combinan, tiende a acercase al URS, en las dos comunidades de estudio.

La inversión en conservación de suelos a largo plazo incrementa los contenidos de materia orgánica en los suelos y es reconocida por los productores como una acción estratégica para aumentar la producción agraria de manera sostenible.

Finalmente corroboramos que los productores de ambas comunidades que adoptan técnicas de conservación de suelo y agua mejoran sus suelos e ingresos.

No solo basta transferir tecnologías para llegar a una adopción, el éxito se fundamenta en la estrategia comunicacional y motivación que tiene el extensionista para lograr los verdaderos procesos de cambios.

\section{REFERENCIAS BIBLIOGRÁFICAS}

Barreto, H. 1996. Atlas digital de Nicaragua. Centro Internacional de Agricultura Tropical. Laderas Proyecto. Tegucigalpa, HN. p 5. Hernández, JC; Araya, R. 2004. Logros en la implementación del fitomejoramiento participativo (FP) en frijol en Costa Rica. In: VIII Reunión anual del sector frijolero de Costa Rica. Agosto 2003. San Carlos, CR. p 3-24.

INIFOM (Instituto Nicaragüense de Fomento Municipal). 2000. Caracterizaciones municipales de Nicaragua.

IICA (Instituto Interamericano de Cooperación para la Agricultura, NI). 2005. Inventario de políticas agroambientales en Nicaragua, descripción de los principales problemas ambientales relacionado con la agricultura. Tecnología. Managua NI. p 6- 8 .

Kononova, MM. 1981. Materia orgánica del suelo. Ed. Oikos. Barcelona.

PASOLAC (Programa de Agricultura Sostenible en Laderas de América Central). 2003. Informe anual regional. Los pequeños y medianos productores que adoptan tecnología de manejo sostenible de suelo y agua mejoran sus ingresos y el empleo rural. Managua, Tegucigalpa y San Salvador. p 8.

. 1999. Guía técnica de conservación de suelo y agua. Managua NI.

Rogers, E. 1995. Diffusion of innovations. 4 ed. New York, US. The Free Press. 519 p.

Reyes, J; Arauz, K. 2009. Factores que influyen en la adopción de tecnologías de conservación de suelos y agua en productores de la comunidad de Tomabú, municipio de La Trinidad, departamento de Estelí en el período 2002-2006. Managua NI. 62 p.

UNA (Universidad Nacional Agraria, NI). 2008. Guías y normas de las formas de culminación de estudios. Mangua, NI. 56 p. 